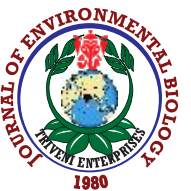

\title{
Nanomaterial's toxicity and its regulation strategies
}

\author{
S. Naqvi and S.J.S. Flora* \\ Department of Pharmacology and Toxicology, National Institute of Pharmaceutical Education and Research, Lucknow - 226002 , India \\ *Corresponding Author Email : sjsflora@hotmail.com
}

Paper received: 13.01 .2019

Revised received: 18.03 .2020

Accepted: 04.04 .2020

\begin{abstract}
Nanotechnology is having a great deal of public interest, due to its wide array of applications in biomedical research including drug delivery, biosensors, imaging, stem cells, regenerative medicines and other industries such as agriculture, solar energy, cosmetics, etc. The increasing and demanding role of nanomaterials also poses a great challenge to their end-users, their unique physio-chemical properties from their bulk counterpart make them interestingly novel but raise a matter of concern in terms of toxicity. Reliable characterization of nanomaterials and their mechanism of action, their final fate and, behavior in the environment and human body are still not fully understood or explored well. The legal framework adopted by various regulatory bodies for handling nanomaterials before coming to the marketplace should be robust and flexible and should reflect the characteristics of specific products, and provide a clear idea of the issues related to their safety, efficacy, public health impact and their final fate in the environment. In the current review, toxicity of nanomaterials and their risk assessment strategies by various regulatory bodies is discussed.
\end{abstract}

\section{Introduction}

Nanotechnology is a multidisciplinary branch of science, engineering and technology conducted at nanoscale. It has grown in to a multibillion-dollar industry all over the world with hundreds of products enabled by nanotechnology for commercial use. Currently, nanotechnology is applied in the field of medicine, environmental protection, cosmetics, energy, and space exploration. It has attracted a great deal of public interest compared to bulk compounds and helped in improving the human needs. Nanotechnology besides wide applications and has revolutionized industrial sector particularly information technology and health care sector (Quesada-González et al., 2018). It provides several useful applications in highly sophisticated technologies due to their unique physical and chemical properties compared to their respective bulk compounds. However, these unique and specific properties of nanomaterials may pose toxic effects on the ecosystem, aquatic system, health, and environment (Gracia-Gomex et al., 2020, Environmental exposure to nanomaterials has grown significantly over the past decades as they have become part of our daily life (Bakshi et al., 2020). Hence, studies investigating the magnitude of release of nanomaterials and their subsequent environmental fate, transport, transformation, and potential for human and environmental exposure and their sources of toxicity, disposal of various classes of nanomaterials are still lacking, therefore toxicity also increases day by day and, as a result, nanotoxicity research is gaining a lot of attention and responsiveness, hence current days it is the matter of priority to resolve the limitations and the issues created by nanomaterials. Both benefits and implications of nano-science and nanotechnology on environmental and human health depend on their sustainable

How to cite : Naqvi, S. and S.J.S. Flora: Nanomaterial's toxicity and its regulation strategies. J. Environ. Biol., 41, 659-671 (2020). 
design and development. There is always a combination of potential, promise and uncertainty with new emerging technologies and our regulatory agencies deal with all these aspects before launching a product in the market (Wolfram et al., 2015, Clausan et al., 2018, Naqvi et al., 2018, Li et al., 2020). This review discusses a brief description of regulations, risk assessment strategies of nanomaterials present in the environment.

Nanomaterials : Nanomaterials can be defined as any organic, inorganic, or organometallic substances which show different chemical, physical, and/or electrical properties and can change as a function of size and shape and partially characterized by their miniature size, conducted at nanoscale and can be measured in nanometres. A nanometer is approximately 100,000 times smaller than the diameter of a human hair. Fig. 1 demonstrates the size of materials that exist in nature at nanoscale level (https://introtonanotechnology.weebly.com/the-nanoscale).

Nanomaterials already exist in nature, and they are classified on the basic of their nature of origin. Some are engineered nanomaterials like polymeric, liposomal, and carbon nanotubes. On the other hand, some are naturally occurring nanomaterials produced unintentionally from nature like mineral aerosol from wind erosion of soil, sea salt, dust, evaporation, ash and smoke particulates from forest fires. Incidental nanomaterials are ultrafine particulate matter obtained from automobile exhaust, carbon-based nanomaterials, metal or metal-oxide nanoparticles, and quantum dots. $\mathrm{ZnO}$ nanorods and nanoanions exhibit nanoscale properties. Currently, NM's are used for manufacturing polymers based nanoparticles at molecular level, solar cells, computer chip, pharmaceutical packaging market.
Nowadays, scientists and researchers are particularly showing interest in engineered nanomaterials (ENMs), which are manufactured and fabricated for application in various industrial and medical sectors, electrical and electronic. Currently interest is growing among the researchers for engineered nanomaterials (ENMs), which are manufactured and fabricated for applications in various industrial and medical sectors, electrical and electronic, energy, cosmetics, sunscreens, sporting goods, clothing and food packaging etc (Bumbudsanpharoke et al. 2015). In addition, they are widely employed in medical diagnosis (Sharma et al., 2019), imaging, gene delivery (Panghal et al., 2018; Naqvi et al., 2012) and drug delivery, therapeutics (Kushwaha et al.,2018; Muhiyuddin et al., 2017, Naqvi et al., 2018; Yadav et. al., 2014, 2016, 2018). However, stringent regulation and dynamic regulatory measures by governing bodies are the major constrainson suppliers, manufactures of counterfeit medicines. Yadav et al. (2012) prepared curcumin loaded chitosan nanoparticles and used it as a strong detoxifying agent against arsenic poisoning. Co-administration of encapsulated curcumin nanoparticles (ECNPs) had pronounced therapeutic effects on oxidative stress parameters, i.e., blood $\delta$-aminolevulinic acid dehydratase activity, glutathione and blood reactive oxygen species induced by arsenic. The authors reported that ECNPs exhibit improved antioxidant and chelating potential (dose $1.5 \mathrm{mg}$ $\mathrm{kg}^{-1}$ ) as compared to $15 \mathrm{mg} \mathrm{kg}^{-1}$ dose of free curcumin based on the observation like better neuroprotective efficacy compared to bulk curcumin. Naqvi et al. (2019) recently tested the therapeutic potential of lactobionic acid conjugated quercetin loaded organically modified silica nanoparticles and observed significant hepatoprotective effect compared to bulk quercetin against cyclophosphamide induced hepatotoxicity.
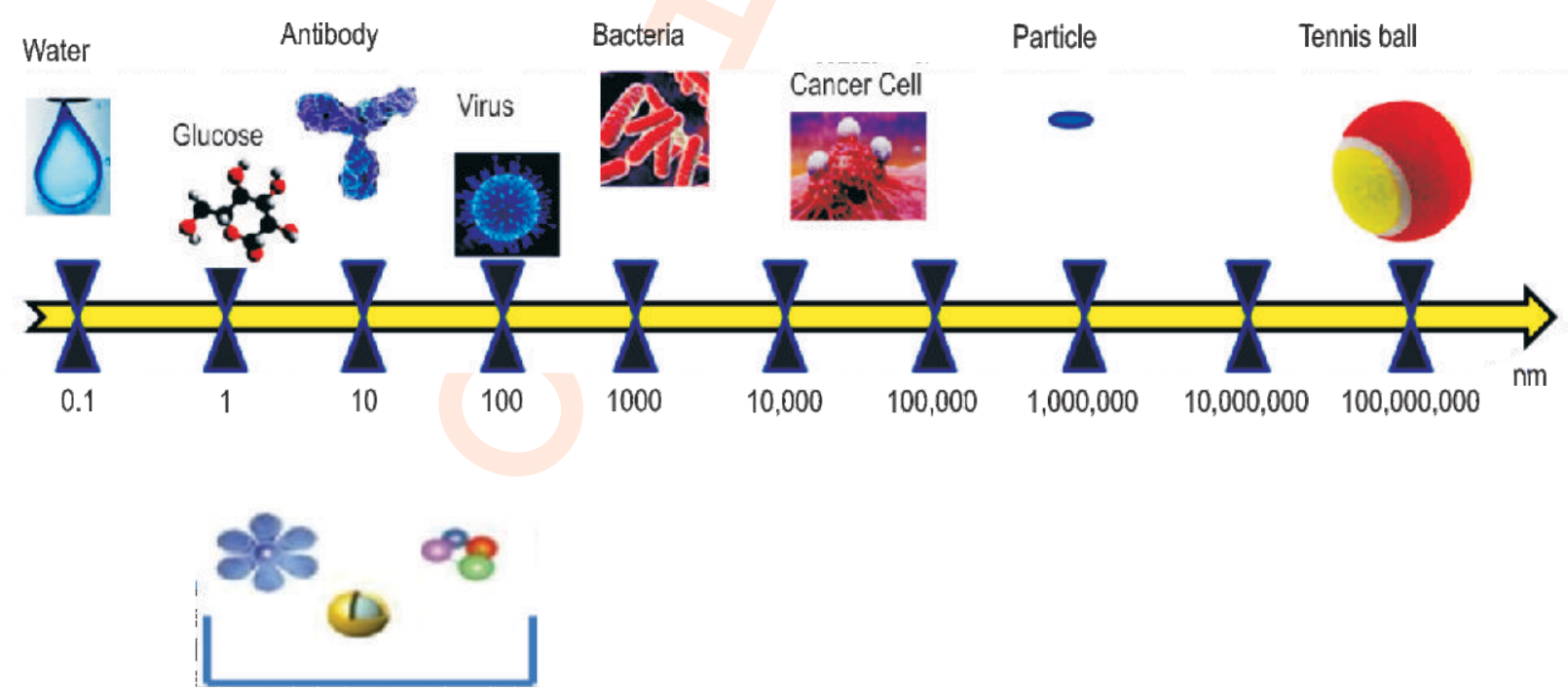

Nanodevices:

Nanopores, Dendrimers, Nanotubes, Quantum dots and Nanoshell

Fig. 1 : Size of materials that exist in nature at nanoscale level. 
Nanotoxicology : It is the branch of science which deals with studying the toxic/adverse effects of nanomaterials on living organisms and environment, and explains the possible hazardous effects of nanomaterials/nanoparticles having diameter less than 100 nanometers. Nanotoxicology deals with classifying the conditions that have toxic effects and determining their unique properties of specific nanoscale particles on both public health and environment. The primary goal of nanotoxicology is to identify and characterize the potential hazards to human and environment associated with the use of ENMs for risk assessment. It, thus, requires multidisciplinary approach involving various disciplines like biology (including toxicology), chemistry, physics, geology, physiology (pharmacodynamics and pharmacokinetics). Exposure assessment and fate transport modelling of nanomaterials is also an important component of this subject for developing and implementing testing strategies, establishing toxicity ranking in order to conduct 'safe' exposure levels and preventive exposure guidelines. Fig. 2 demonstrates how nanoparticles such as $\mathrm{TiO}_{2}$, $\mathrm{Cu}, \mathrm{Ag}$, graphene, and other carbon based nanomaterials enter the cell membrane and depending on their shape, size and dose they cause either therapeutic effects or deleterious effects such as mitochondrial damage, cell cycle arrest, DNA damage, enzyme disruption, apoptosis, and cell membrane damage (Yuan et al., 2019). Toxicity of platinum nanoparticles of size 30-60nm was evaluated by Alshatwi et al. (2015) in SiHa cells (a cervical cancer cell line) for 24 hrs. Analysis of cell cycle, nuclear morphology and cell cytotoxicity showed nuclear fragmentation, chromatin condensation, and nuclear swelling in cells. Agglomeration is the one of the key factors for determining the risk of nanomaterials toxicity. Murugadoss et al. (2020) studied the cytotoxicity, glutathione metabolism, epithelial barrier integrity, inflammatory mediators and DNA damage following exposure to $\mathrm{TiO}_{2}$ nanoparticles of two different sizes (17 and $117 \mathrm{~nm}$ ) in human bronchial epithelial, colon epithelial and monocytic (THP-1) cell lines. Exposure to large agglomerates of $\mathrm{TiO}_{2}$ led to enhanced glutathione depletion, DNA damage and higher levels of IL-8 and IL-1 in THP-1 compared to small agglomerates. No effect of agglomeration was noticed with 117 $\mathrm{nm} \mathrm{TiO}_{2}$. Oral administration of $\mathrm{TiO}_{2}$ suspension in $\mathrm{C} 57 \mathrm{BL} / 6 \mathrm{JRj}$ mice resulted in more pronounced pulmonary responses, DNA damage in aspirated mice exposed to large agglomerates of 117 $\mathrm{nm} \mathrm{TiO}_{2}$ compared to small agglomerates. Toxicity of nanomaterials is mediated by oxidative stress leading to pronounced immuno- and genotoxicity. Fig. 3 demonstrates the hazards associated with organ toxicity on exposure to nanoparticles. Sub-chronic inhalation study was carried out to determine the effects of lead and lead oxide nanoparticles. Mice were exposed for 2, 5 and 13 weeks to lead and lead oxide nanoparticles $\left(192.5 \mathrm{\mu g} \mathrm{m}^{-3}\right)$ to monitor their distribution in various soft tissues like lung, liver, kidney and brain. The results of different biomarkers of DNA damage, i.e., hydroxylated deoxoguanosine (8-OHdG) and lipid damage F2-isoprostanes (8-iso-prostaglandins $F_{\text {2-apha }}$ and $E_{2}$ ) and thiobarbituric acid reactive species (TBARS) were demonstrated. Fast blood transport and distribution of lead from lung to kidneys and liver. Lead enters the brain via nasal nerve to olfactory bulbs. High levels of TBARS and isoprostanes have been reported in brain on long term exposure. Long term exposure to lead oxide nanoparticles may pose high risk on human health and environment (Blahova et al., 2020).

There is an urgent need to evaluate the mechanism involved for toxicity pathways like epigenetic alterations, and conventional toxicity testing methods. To initiate epigenetic, toxicity screening of different engineered nanomaterials, better understanding is required to establish the links between exposure, epigenetic changes and adverse phenotypic endpoints, which will lead to the development of better mechanistic approach to monitor epigenetic toxicity (Dusinska et al., 2017).

Generally, the characterization of nanomaterials depends on their behaviour and toxicity which are subjective to their physico-chemical characteristics. The toxic characteristics can be determined by evaluating their nanosize properties (shape, size, phase, electronic structure, density, temperature and crystalline) and surface area characteristics (surface electronic structure, surface functionality), reactivity, surface charge, surface chemistry, unique quantum and magnetic properties, geometry and dimensions, composition and aggregationdependent interactions of nanostructures with biological systems, however, bio persistence is still lacking and unclear to characterize the unintended and undesirable effects of nanomaterials to ensure safety of new therapies and medical devices. Nanotoxicity studies are proposed to define whether and to what extent the properties of nanomaterials in the nanoscale dimensions may pose a menace to the environment and living system. Once NMs are released into the environment, several changes occur in view of the environmental and physicochemical factors like light irradiation, natural organic matter (NOM), ionic strength, co-existing contaminants, temperature, and chemical surface modifications influences the characteristic properties and toxicity and fate of NPs the data obtained from studies help to correlate to chemical, ecological, or biological responses.

Regulations of engineered nanomaterials : The usage of nanomaterials in daily lifestyle has been increasing tremendously in today's technological world. The regulatory and legislative bodies are supposed to account for their safe usage and successful risk assessment. The safety of manufactured nanomaterials is a critical issue and of great concern for lawmakers all over the world. In view of its unique properties like nanosize range, high surface area, catalytic properties they require additional testing beyond guidelines set for toxicity test. To ensure safety of nanomaterials containing products, several 

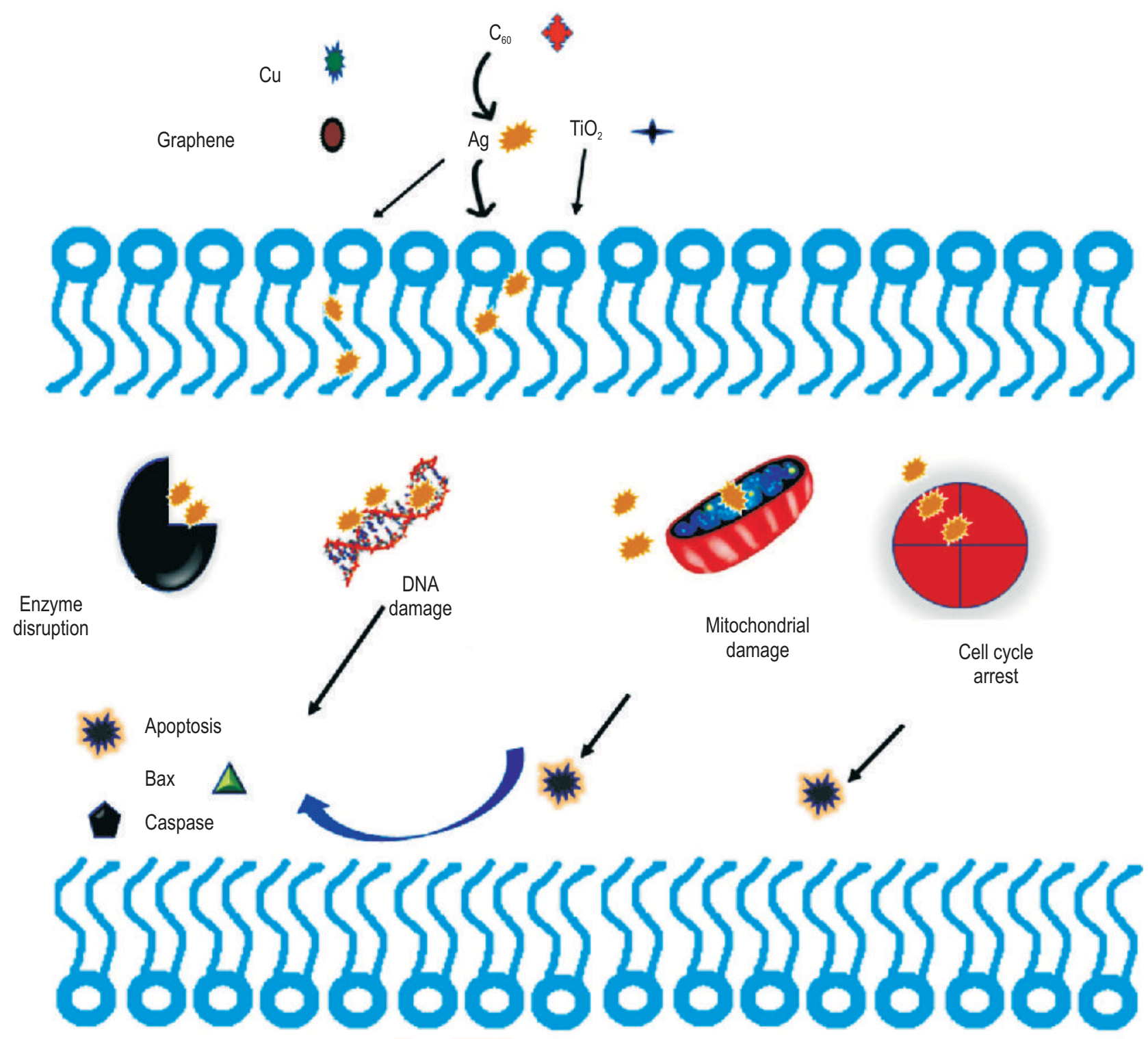

Fig. 2 : Schematic illustration of damage associated at cellular level on exposure to nanomaterials.

national and international regulatory bodies like ISO their safety before launching in the market consumers, several national and international regulatory bodies like ISO, European Committee for Standardization and the Organization for Economic Cooperation and Development are focussing on the nomenclature and definitions, test methods to characterize and recognize the toxicity of nanoparticles and safety potential, protocols for toxicity, standard test methods, health, and safety concern as well as environmental-related facet. These standard regulatory bodies create a framework to set up working groups of nanotechnology and safe use of nanomaterials, and their related work in cooperation on issues to avoid duplicate testing and make sure that their nanotechnology output is complementary. The short term goal (ISO et al., 2018) is to develop standards for protocols for pharmacokinetics, defining a minimum set of measurements, particle characterization protocols for nano-health, toxicology screening tests, methods to determine stability of nanomaterials in animal models and standards for drug delivery and the route of exposure to nanomaterials.

Toxic Substances Control Act (TSCA) reforms legislation and regulation for chemicals testing. Chemical manufacturers and companies using nanomaterials as the end products need to be vigilant and follow TSCA guidelines during their manufacturing process. Failure to implement regulatory requirements might result in business interruption in a company and leading to customers' dissatisfaction.

In 2007, OECD along with their Working Partners (government, industry, academia) launched the Sponsorship 


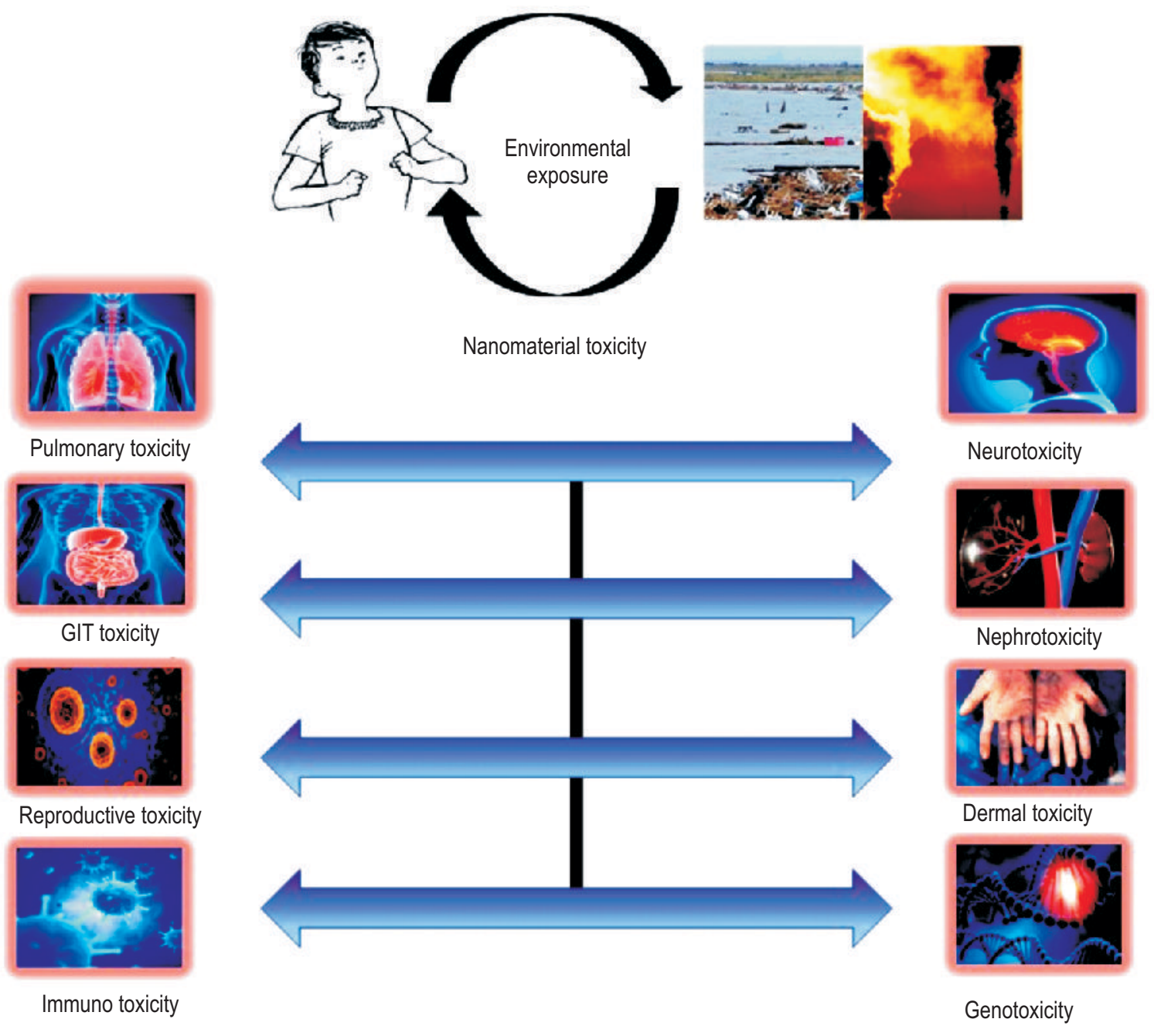

Fig. 3 : Health hazards associated with organ toxicity upon exposure to nanoparticles.

Programme for testing dossiers of eleven Manufactured Nanomaterials, including cerium oxide, dendrimers, fullerenes, gold, silver nanoparticles, carbon nanotubes (MWCNTs, SWCNTs), nanoclays, $\mathrm{SiO}_{2}, \mathrm{TiO}_{2}, \mathrm{ZnO}$. Their goal is to provide scientific endpoint data, relevant to human health and environmental safety, by conducting specific tests and providing No Observed Adverse Effect Level (NOAEL), Low Observed Adverse Effect Level (LOAEL), Lethal concentration $\left(\mathrm{LC}_{50}\right)$, Lethal Dose $\left(\mathrm{LD}_{50}\right)$ in animal and zebrafish models. Testing program aims to assess the existing guidelines for nanomaterials as well as to compare the unique properties of nanomaterials to their bulk counterparts. All physico-chemical properties of nanomaterials are tested against bulk material which provide strategies for the identification of hazard, risk assessment or risk management measures for future use and safety concerns of manufactured nanomaterials.

Environmental Protection Agency (EPA) under the Toxic Substances Control Act (TSCA) and Federal Insecticide, Fungicide, and Rodenticide Act (FIFRA) are two agencies that regulate nanomaterials in the USA. Other US Federal Government regulatory agencies, like Food and Drug Administration (FDA), also regulates nanomaterials, in collaboration with EPA to check the progress of nanomaterials in different categories of nanoproduction, such as cosmetics, medical devices, drugs and food. The EPA has revised and reconstructs its amendments or legislations towards the regulation of nanomaterials. Earlier manufacturers of nanomaterials were supposed to share information on nanomaterials through Nanoscale Materials Stewardship Program (NMSP) voluntarily. EPA however shifted towards mandatory laws to collect complete information regarding the set of standards on production, usage and safe disposal of nanomaterials, with mandatory pre-manufacture details for new nanomaterials. The laws are stringent for collection of both existing and new nanomaterials (Duvall et al., 2011).

TSCA pursue some specific facts on new chemical substances to revise the risk of nanomaterials on environment 


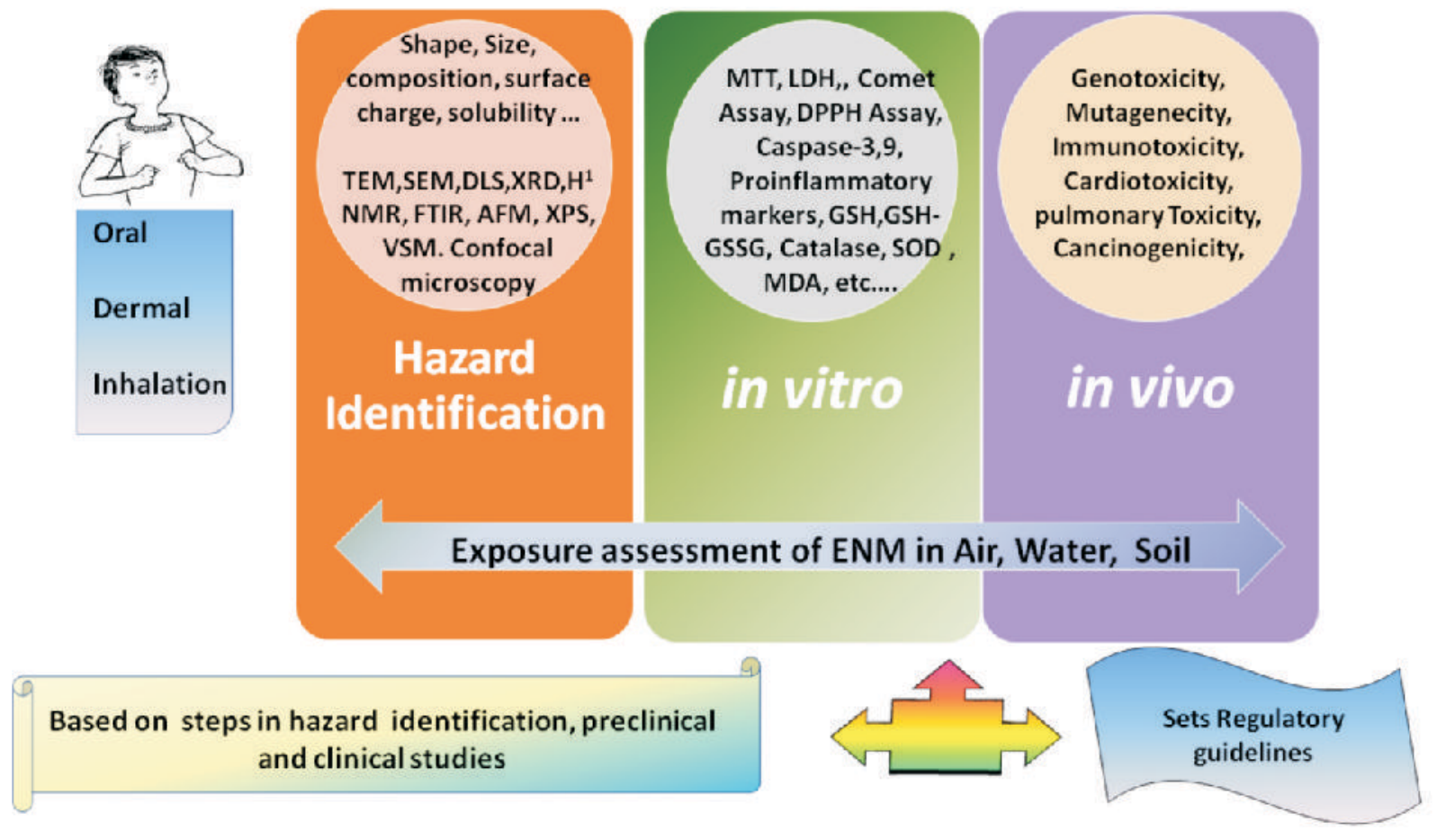

Fig. 4 : Schematic illustration of approaches towards risk assessment of nanomaterials.

and human health, before manufacturing nanomaterials under Pre-manufacture Notification Rule (Duvall et al., 2011). Recently, the regulatory agencies have taken steps to control the massive use of nanomaterials, like limiting the, use of personal protective equipment (PPE), reduce the release and entry of nanomaterials into the environment and ecosystem and need for testing to generate raw data regarding environmental and health effects. Currently, the utilization of the nanoscale materials by the manufacturers is controlled under consent order or Significant New Use Rules (SNUR) under TSCA.

Pesticides which are of nanoscale range, the U.S Environmental Protection Agency (EPA) deals them under FIFRA Act. Nanomaterials used for cure mitigation or prevention of pests and microorganisms are regulated under the Federal Insecticide, Fungicide and Rodenticide Act (FIFRA), is working on modifications of pesticide registration guidelines to know the composition of nanomaterials present in the pesticides. Similar to EPA, FDA also regulates nanomaterials present in different classes like cosmetics, food and medicinal products. Number of industrial guidelines published by FDA is currently available for controlling and safe use of nanomaterials in cosmetics, food ingredients and food contact substances and colour additives in food (Duvall et al., 2011).

The European Commission has set rules and regulations for nanomaterials manufacturers, before launching them in the market and further use by consumers. 'Registration, Evaluation, Authorization and Restriction of Chemicals' (REACH) and
Classification, Labelling and Packaging of Substances and Mixtures (CLP) regulatory bodies of the European Union which aims to provide better protection to human health and environment and manage risk to products/substances// nanomaterials by evaluating their intrinsic properties. Manufacturers and importers have to submit their scientific data to the European Chemical Agency (ECHA) in Helsinki. In-depth evaluation of suspicious nanomaterials should be done extensively by agency and provide safety information to the manufacturers and consumers. They follow the four-tiered process: Registration; Evaluation; Authorization; Restriction of Chemicals. "Second Regulatory Review on Nanomaterials" and the "Commission Staff Working Document" contains all the required information on nanomaterials regulation mentioned in the Commission Communication. Information on nano technologies, in general, is available on the Europa website on nanotechnologies as well as on the European Observatory for Nanomaterials (EUON) (FDA web link; ECHA web link). France under the Grenelle II Act and Austria under Nanotechnology Action Plan have issued mandatory reporting and tracking systems for the safety of nanomaterials.

Currently, as per Canadian Government the nanosubstances that are not listed under Domestic Substances List (DSL) are subjected to New Substances Notification Regulations (Chemicals and Polymers) under Canadian Environmental Protection Act (CEPA), 1999. Nanoportal is designed by the Canadian Government and it provides information in nanotechnology programs. A nanomaterial already listed in DSL 
has not been considered in risk assessments of existing substances conducted under CEPA (1999). The Government of Canada wants to ensure that nanomaterials currently in commerce in Canada may require further action to determine if they pose any potential risks to the environment or human health among existing nanomaterials (nanoportal web link).

Several steps have been taken in setting nano regulation in countries like China, Korea, Japan, India, Iran, etc. Regulation for nanomaterials is still underway in Asian countries compared to US and European countries. However, specific regulation for all types, shape and size of nanomaterials are not yet finalized by any regulatory bodies like EU and USA. In India, Department of Science and Technology, Department of Biotechnology has drafted a Guideline on the "Evaluation of Nano-Agriinput Products and Nano-Agriproducts" in 2019, where they define and characteriz various nanomaterials used in agriproducts based on their chemical nature (organic, inorganic, composite); degradation nature (Biodegradable and Non-biodegradable); nanocarriers loaded with active ingredient and active ingredient converted to nanoform and on the nature based on the their synthesis, i.e., biological, chemical and physical (dbtindia.gov.inweblink). Department of Science and Technology (DST), Government of India (with inputs from Nanoregulatory Task Force) has constituted group of eminent experts working in nanotechnology and out a comprehensive document on "Guidelines and Best Practices for Safe Handling of Nanomaterials in Research Laboratories and Industries"(dst.gov.in-weblink).

The Ministry of Health, Labour and Welfare (MHLW) of Japan has encouraged companies to take necessary precautions while manufacturing nanomaterials. Nanomaterial's safety issues are under progress by the Ministry of Health, Labour and Welfare (MHLW) and Ministry of the Environment (MOE) in Japan. The National Institute of Advanced Industrial Science and Technology (AIST), of Japan focuses on the creation and practical realization of technologies which are useful to Japanese industry and society, and on "bridging" the gap between innovative technological seeds and commercialization. AIST has recently published few reports on the risk assessment of manufactured nanomaterials, such as fullerenes, carbon nanotubes, and $\mathrm{TiO} 2$ in effort to make regulations on nanomaterials (Birch et al., 2011). Some federal regulatory agencies provide information regarding nanotechnology includes: The U.S. Food and Drug Administration (FDA) regulate a broad array of product range such as foods, cosmetics, drugs, devices, and veterinary products. Many nanomaterials are dealt under the Toxic Substances Control Act (TSCA) under U.S. Environmental Protection Agency (EPA). The U.S. Consumer Product Safety Commission (CPSC) is an independent federal regulatory agency, formed in 1972 by Congress in the Consumer Product Safety Act. In that law, Congress directed the CPSC to "protect the public against unreasonable risks of injuries and deaths associated with consumer products." National Institute of Environmental Health Sciences (NIEHS) has taken the initiative as control measures for the Health and Safety of nanomaterials exposure. Under the National Nanotechnology Initiative Program, they launched "ONE Nano". Under this strategic program, they assess their safety and project the predictive models for quantification of nanomaterials, after their Adsorption, distribution, and metabolism inside living organism and further gives idea and guidelines for safe and efficient designing and synthesis of engineered nanomaterials for future use. The National Nanotechnology Initiative (NNI), a federal, multi-agency program of United States committed to accelerating high end and translational nanotechnology research for development, to promote the transfer of new and advance technology to the public domain. The Nanoscale Science, Engineering and Technology (NSET) subcommittee of NNI has four working groups that harmonize the planning, budget, program implementation and review the nanotechnology initiative. The Nanotechnology Environmental and Health Implications (NEHI) subcommittee are supporting Federal activities focused on the health and safety implications of nanotechnology-related products. National Institute of Environmental Health Sciences (NIEHS) provide support to improve public health, aligned with two $\mathrm{NIH}$ institutes, i.e., National Institute of Biomedical Imaging and Bioengineering (NIBIB) and the National Cancer Institute (NCI) and develop NanoRegistry where they recommend a central repository of nanotechnology-related published literature to the public domain. NIEHS is working to understand the health effects of human exposure to nanomaterials. It promotes an interagency agreement with NCl's Nanotechnology Characterization Laboratory to provide NIEHS grantees common engineered nanomaterials (ENMs) and to characterize the physical and chemical properties. The NIH Nanomedicine Initiative is a crossinstitute attempt to comprehend and develop nanoscale technologies that can be useful for therapeutic strategies. The NIH "Nano Task Force", coordinated by NIEHS embodies the safety and toxicology interests of institutional bodies within $\mathrm{NIH}$ to understand medical use to develop novel technologies and products.

Strategies for environmental regulations for nanomaterials risk assessment : The present assessments for the legislation and regulation of nano base products are not satisfactory to deal with the hazards associated with it, because of its unique properties, their potential threats to the aquatic environment, ecosystem and public health are high. However, the regulatory bodies in different countries are keenly playing a part to resolve the risks and issues associated with nanomaterials. Recently, the National Nanotechnology Initiatives (NNI) of the US government realizes the potential benefits and risks of nanomaterials and projected \$2.1 and further increased to \$201 million. The predicted global market value of engineered nanomaterials (ENM's) have potential to reach upto 11.8 billion US\$ by the year 2025 (Mordor Intelligence, 2016). 
Studies have shown nanoparticles with low solubility are more toxic than larger particles (Sharifi et al., 2012). Hence, some indications proved that physicochemical properties like particle size, surface area, and surface chemistry are responsible for observed responses in both in vitro (cell cultures) and in vivo (animals). A study suggests that some nanoparticles can travel from the respiratory system to other body organs. Research is still going on to understand how these unique characteristic properties of nanomaterials have a wide impact on environmental exposure (Nanoecotoxicology) and may lead to specific health effects.

Engineered nanomaterials based approaches have been widely investigated in a technologically interesting domain ranging from drug delivery, diagnostics, advanced therapeutics and leads to huge global commercial escalation and future expectations for industry such as sensors, electronics, pharma, energy, water, textile, and cosmetics. There is no doubt that nanotechnology has revolutionized the novel approaches for therapy, especially cancer and several other human diseases such as gene therapy and lifestyle diseases. The US Food and Drug Administration (FDA) has approved Paclitaxel (Abraxane) and Doxorubicin (Doxil) for clinical use and many more drugs are currently in clinical trials. Currently, engineered nanomaterials are used in more than eight hundreds consumer products (Maynard et al., 2004), including electronic, cosmetics (sunscreens, shampoos), cigarette filters, fabric, automotives, agriculture, antimicrobial, medical and pharmaceuticals etc. Apart from positive role in human health and development, it has some potential health hazards but this can be overcomed by systemic risk assessment in terms of safety as well as benefits before its implication to end-users.

Nanomaterials and their toxic potentials have received significant attention by nanotoxicologist, nanomaterials such as polymeric, metallic, carbon based nanomaterials nanotubes, and organic or inorganic are omnipresent and are used daily as lifestyle products. They are present in body lotions, shampoos, toothpaste, paint, antiperspirants, sunscreen etc. However, the risk assessment of nanomaterials is quite challenging, because, after disposal, nanomaterials are released in water, plants, sludge, and soil. The accurate measure of nanomaterials in the environment after its disposal is critical as it is very difficult to determine how these nanomaterials and engineered nanomaterials behave in different substrates and exhibit potential damage. Currently, the risk management of nanomaterials is significantly affected due to inadequate knowledge of their physio-chemical properties, their safety profile, toxicity and benefits of different product classes and their different applications in diverse fields. Elements like arsenic, lead, mercury, cadmium, selenium and sodium tungstate are toxic in their bulk form (Agarwal et al., 2015; Flora et al., 2013; Mittal et al., 2018; Jain et al., 2012, 2014; Sachdeva et al., 2015; Srivastava et al., 2010), however, it is not necessary that all nanomaterials and their bulk materials are toxic in nature, their dose and concentration, size and shape is a decisive factor in determining their toxicity (Naqvi et al., 2010; Chouhan et al., 2013).

Unique feature of nanomaterials at nanoscale dimensions, from their bulk counterparts such as high surface area to mass ratio, nanosize range and morphology, high catalytic and optical properties, new and altered physico-chemical properties such as melting point/solubility, electrical conductivity, amorphous and crystalline (Pichardo et al.,2012; Maynard et al., 2007; Yang et al., 2009; Peters et al., 2009) enable them to develop novel products, but simultaneously make their risk management strategies difficult because their behaviour and fate varies in different compartment of the environment. On the other hand, their unique characteristics, make them promising for technological application, concurrently also affect human wellbeing, and produce cytotoxic and mutagenic effects, pulmonary, cardiotoxic, dermatologic, musculoskeletal, neurotoxicity, reproductive effects, and inflammation. In risk assessment, hazard identification is very critical point, what, where and when, individual or subject and population, this should be dealt carefully, and then only dose-response relationship can be established successfully. The quantitative assessment of released nanomaterials in the environmental compartment, i.e., air, water, soil, and the organism is highly requisite. However, it is unfortunate, to date, not much is apparent from analytical data (e.g. actual concentration of released and exposed nanomaterials in the environment) nor with respect to theoretical or modelling studies. Few initiatives should be taken on priority during risk assessment of nanomaterials which minimizes or eliminates toxicity to the end-users.

In hazard identification, the potential nanomaterials are identified initially by evaluating their specific physico-chemical behaviour upon release into the environment. Their physical and chemical properties includes dissolution/speciation, level of agglomeration/aggregation, $\mathrm{pH}$, shape morphology, size, chemical composition, surface chemistry, water-solubility, partition coefficient, oxidation reduction potential, radical formation potential, catalytic activity and interaction with surrounding media (air, water, soil or organisms) (Naqvi et al., 2018). Understanding the properties of nanomaterials is a decisive factor for selecting right strategies for hazard identification, risk assessment or risk management measures. Firstly, characterization of nanomaterials should be done for understanding their properties by various standard techniques such as shape and size is determined by Transmission electron microscopy (TEM), for stability of nanomaterials, surface charge is measured by zeta potential in the aqueous phase. Surface morphology by scanning microscope (SEM), chemical group interaction by Fourier transform Infrared spectroscopy (FTIR), Raman spectroscopy, Nuclear magnetic resonance (NMR), 
crystalline/amorphous behaviour by X-Ray diffraction (XRD), atomic force microscopy (AFM), confocal microscopy, optical and fluorescence microscopy, Surface-enhanced Raman spectroscopy (SERS), Inductively Coupled Plasma Mass Spectrometry (ICP-MS), Inductively Coupled Plasma Optical Emission Spectroscopy (ICP-OES), and Energy-dispersive X-ray spectroscopy (EDS) is used to investigate the elemental composition of nanomaterials, Brunauer-Emmet-Teller (BET), nanoparticles tracking analysis (NTA), and small angle neutron scattering (SANS). Different assays are reported in preclinical and clinical studies to evaluate cytotoxicity/biocompatibility. Immunotoxicity analysis is often evaluated by enzyme-linked immunosorbent assay (ELISA) Secondly, hazard characterization, i.e., dose-response relationship, and their environmental fate. The toxicological and ecotoxicological effects are evaluated by understanding the mechanism of toxicity as endpoints, i.e., organ toxicity (Liver, CNS, lungs, kidney, cardiovascular, reproductive toxicity, nephrotoxicity, immunotoxicity) in mammalian and human subjects. The determination of real exposure concentration is the major hurdle in nanomaterials toxicity assessment. Two Major issues should be dealt carefully for determining the toxicity in environment and human body (a) solubility of nanomaterials (b) suitable analytical techniques for their characterization. The route of exposure of nanomaterials is also an important parameter of toxicity. It enters human beings via inhalation, oral and dermal route. After physicochemical characterization of nanomaterials, in vitro and in-vivo toxicity studies should be carried out for hazard identification process. The in vitro studies in different cell lines like liver, cancer, macrophages (Chang et al., 2014) neuronal, pancreas gives the basic idea and screen the compounds for in- vivo toxicity. The chemical and ecotoxicological assessment can be done in the zebrafish model (Lowry et al., 2012). Cell metabolic activity measure by different cytotoxic assays such as colorimetric assay (MTT, Alamar blue, XTT), lactate dehydrogenase assay (LDH) (Flora, 2017; Naqvi et al., 2010), helps in determining the index of intrinsic cytotoxicity. Silver and gold nanoparticles have been used extensively for wide applications such as biosensors, drug delivery, bioimaging, shampoos, cosmetics and cancer. Srivastava et al. (2016) studied the effect of Ag and Au NPs (1M and $2 \mathrm{M}$, orally) for fourteen consecutive days. They observed hepatic and renal toxicity, as well as enhanced interleukin- 6 and nitric oxide synthase levels at given dose. A significant increase was observed in kidney Metallothionein (MT) in contrast to liver MT, it was further confirmed by DNA damage in urine samples by increased 8-hydroxy-20-deoxyguanosine levels. They found that at higher doses i.e., $2 \mathrm{M}, \mathrm{Ag} \mathrm{NP}$ was more toxic as compared to lower dose $(1 \mathrm{M})$. They found that oxidative stress is the chief mechanism accountable for toxic manifestation of silver and gold nanoparticles. The mechanistic data obtained from in-silico and in-vitro studies should be aligned with in-vivo studies, hence it can extrapolate human exposure assessment easily. Moreover, the nanomaterials' ability to permeate various body barriers like blood-brain barrier, blood-placental barrier, blood-testicular barrier, their distribution and circulation in the bloodstream have been tested in in-vivo studies (Maynard et al., 2004). DeLoid et al. (2017) studied in-vitro dosimetry of dispersed, engineered nanomaterials. For risk assessment of engineered nanomaterials accurate dose metrics is an important and critical parameter. Glen et al. (2017) developed and proposed a standardized and integrated methodologies for in-vitro screening by generating ENM suspensions, their colloidal characterization, particularly their kinetics and finally their fate and transport modelling during in-vitro exposure time period.

The assessment of human health risk to various nanomaterials required validated analytical techniques for their accurate detection and measurement in exposed environment. The occupational hazard of engineered nanomaterials are found to be more as compared to other route of environmental exposure, this may be due to high exposure of nanomaterials during handling at their workplaces. Hence, occupational exposure hazards hold the utmost potential for human toxicity. Hence, keeping in mind the various agencies like FDA, OECD, DBT, DST, CEPA tried to quantify known and predicted risks of nanomaterials and allowing risk management in the exposed population to reduce occupational risk hazards.

Environmental toxicity: Nanomaterials obtained from various sources are of different nature like metals, carbon-based nanomaterials, non- metallic, polymeric, biodegradable; nonbiodegradable enters into the environment via diverse routes at different stages, i.e., at the time of manufacture, end product and waste disposal (Walters et al., 2016). Several reports indicate significantly higher concentration of engineered nanomaterials such as $\mathrm{TiO}_{2}$, fullerenes, graphene, nanosilver, zinc oxide, carbon nanotubes, demonstrate potential health hazards on living organisms upon accumulation in air, water and soil compartment after their release. Hazardous exposure of engineered nanomaterials can affect the function and behaviour of aquatic flora and fauna, soil and sediment (Holden et al., 2014; Cledon et al., 2015). However, their environmental fate and persistence, mechanism of uptake by microbes, undesirable toxic effects on physiology, behaviour, and bioaccumulation in living bodies are still not fully explored. Furthermore, scientific data of engineered nanomaterials on ecosystem is inadequate. Nanomaterials enter into water bodies by several routes such as through: direct entry, agrochemicals; soils runoff; aerial deposition; and sewerage treatment plants, chemical discharge from industries etc. In soils, it enters through the end products of remediation technologies, plant pesticides spray; nanomedicines in veterinary products; aerial deposition; from sewage sludge as fertilizer etc. (Holden et al., 2014). Nanomaterials behaviors vary in different compartment of ecosystem i.e. water, soil, and air. Their surface morphology, shape, size, dose, effect of UV light, their ionic strength in water; $\mathrm{pH}$ plays a very critical role in determination of 


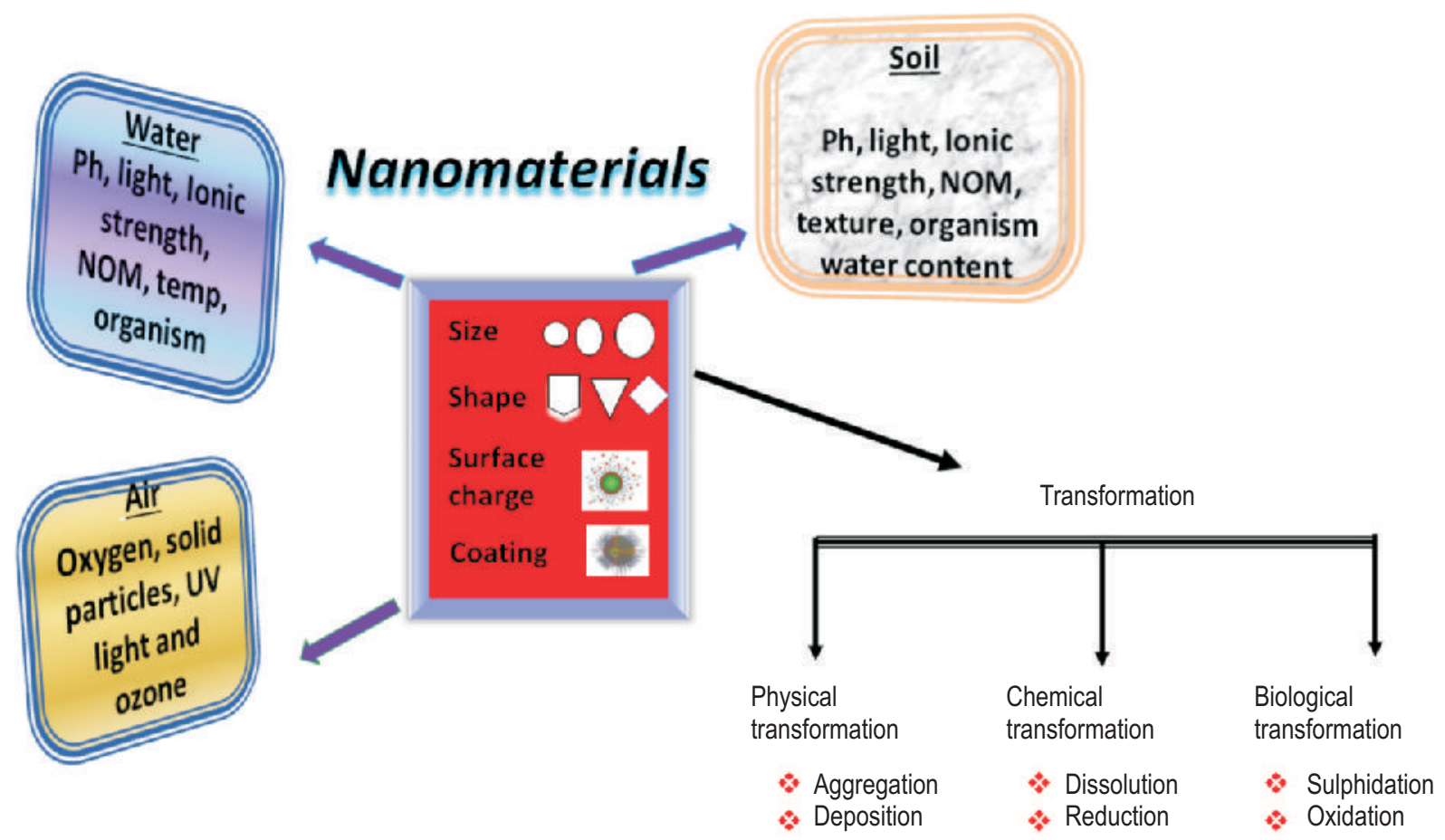

Fig. 5 : Fate and behaviour of engineered nanomaterials in air, water and soil.

toxicity. When it enters into the environment through runoff or other sources, some nanomaterials transform via physical (aggregation, deposition), chemical transformation (dissolution, reduction, oxidation) and biological transformation occurs by a living organism (Stone et al. 2017).

A lot of nanoparticles come in contact with the environment, which ultimately leads to nanoparticulate pollution, via deposition and accumulation of nanoparticles in groundwater and soil for years. There are no reports available to date for the mechanism of their transport, accumulation, biodegradation, agglomeration or dissociation in biological systems to eliminate nanomaterials (Dinesh et al., 2012). Aquatic species (Daphnia and Pimephales) have been studied for checking the toxic manifestations of fullrene-C60; their results demonstrated increased lipid peroxidation levels in the brain, notably augmented LPO in gill, ultimately resulting in enhanced gene expression of inflammatory markers and metabolism. They reported that aggregation occurs in nanoC60, when it comes in contact with water, spontaneously forming stable aggregates (nanoC60) with nanoscale dimensions ( $d=25-500 \mathrm{~nm}$ ). Processes that control transportation and removal of nanomaterials in aquatic, groundwater and wastewater are yet to be investigated to understand the fate of nanoparticles (Dai et al., 2018). Recently, Azhar et al. (2020) demonstrated that ethylene participated in copper oxide nanoparticles (CuO NP)-induced ultrastructural alteration in Arabidopsis thaliana leaves. They observed ethylene-insensitive mutants (ein2-1, ein4 and etr1-3) and WT in response to copper oxide nanoparticles exposure and found augmented oxidative stress and toxicity.

There is always a high risk of transferring nanomaterials from the environment to soil and from soil to consumers. In a recent study, the African giant snail, Achatina fulica was indirectly exposed to nanoplastics from plastic debris in a terrestrial environment from soil via plant. Plants exposed to nanoplastics showed decreased root growth where as snails feeding and foraging speed also decreases. These nanomaterials could have adverse effects directly or indirectly to higher trophic levels (Chae etal., 2020).

There is a drawback in current exposure models which evaluates soluble contaminants released in ecosystems. Initial approaches for assessing suspension's stability, kinetics, dissociation, and aggregation is not much applicable or accurate for nano range materials. (Seetharam et al., 2007; Mackay et al., 2006). Arvidsson et al. (2011) reported that the major drawback of exposure modeling is having a lack of understanding between the interactions of nanomaterials with natural organic matter; hence the current scientific data obtained from experiments is not mimicking the natural environmental conditions.

The fate of nanomaterial's aqueous environment should be monitored via biotic/abiotic processes. Ecological risk assessment is also important to identify the hazardous effects of nanomaterials present in the ecosystem. In addition before launching the physical properties of hazardous and toxic 
nanomaterials such as solubility and degradability should be studied to understand the baseline and detailed information of their safety, toxicity, and dispersion and accumulation in soil and aquatic life in the ecosystem.

Now a days, nanotechnology is the key enabling technology. The potential of nanotechnology-enabled products depend on their safety analysis, efficacy, ultimate fate in the environment, and finally impact public health. Potential toxic endpoints should be thoroughly studied by regulatory bodies as it will help to reveal specific toxicity such as membrane penetration, apoptosis, necrosis, ROS generation, and intracellular accumulation. Although all nanomaterials are not toxic in nature, few nanomaterials (cerium oxide, dendrimers, fullerenes, gold, silver nanoparticles, carbon nanotubes (MWCNTs, SWCNTs), nanoclays, $\mathrm{SiO}_{2}, \mathrm{TiO}_{2}$ (NM100-NM105), ZnO have been declared as safe by regulatory bodies (FDA, OECD, DBT, DST, CEPA). The framework adopted by regulatory bodies should be robust and flexible and should reflect the characteristics of specific products. The regulatory status of engineered nanomaterials or nanotechnology-based products should be addressed case-bycase basis to give transparency to end-users in terms of safety, efficacy, and impact on public health. In conclusion we can say, there is an urgent need for reliable risk assessment techniques, since starting from synthesis of nanomaterials to its end product formation. Further, their final fate and dispersal into the environment should also be critically analyzed. In addition to that, more realistic and accurate exposure set-up which excludes experimental artifact is highly required for analyzing nanomaterials risk-benefit ratio.

\section{Acknowledgments}

SJSF and SN are thankful to the Department of Pharmaceuticals, Ministry of Chemical and Fertilizes, Government of India. S.N. was further supported by Women Scientist Scheme (WOS-A), Department of Science and Technology, Government of India (grant no. SR/WOS-A/LS1224/2015).

\section{References}

Agrawal, S., P. Bhatnagar and S.J.S. Flora: Tissue oxidative stress and changes in the levels of nor-epinephrine and dopamine following co-exposure to lead, arsenic and mercury in rats. Food Chem. Toxicol., 86, 208-216 (2015).

Alshatwi, A.A., J. Athinarayanan and P.V. Subbarayan: Green synthesis of platinum nanoparticles that induce cell death and G2/M-phase cell cycle arrest in human cervical cancer cells. J. Mater. Sci. Med., 26, 5330 (2015). doi:10.1007/s10856-014-5330-1

Arvidson, R., S. Molander, B.A. Sanden and M. Hassellov: Challenges in exposure modelling of nanoparticles in aquatic environments. Hum. Ecol. RiskAssess., 17, 245-262 (2011).

Azhar, W., A.R. Khan, N. Muhammad, B. Liu, G. Song, A. Hussain, M.U. Yasin, S. Khan, R. Munir and Y. Gan: Ethylene mediates CuO NP induced ultrastructural changes and oxidative stress in Arabidopsis thalianaleaves. Environ. Sci. Nano., 7, 938-953(2020)

Bakshi, M.S.: Impact of nanomaterials on ecosystems: Mechanistic aspects in-vivo. Environ Res., 182, 109099 (2020).

Bláhová, L., Z.Nováková, Z. Večeřa, L. Vrlíková, B, Dočekal, J. Dumková, K. Křumal, P. Mikuška, M. Buchtová, A. Hampl, K. Hilscherová and L. Bláha: The effects of nano-sized $\mathrm{PbO}$ on biomarkers of membrane disruption and DNA damage in a subchronic inhalation study on mice. Nanotoxicol., 14, 214-231 (2020).

Birch, M.E., B.K. Ku, D.E. Evans and T.A. Ruda-Eberenz: Exposure and emissions monitoring during carbon nanofiber production- Part l: elemental carbon and iron-soot aerosols. The Annal. Occupat. Hyg., 55, 1016-1136 (2011)

Bumbudsanpharoke, N. and S. Ko: Nano-food packaging:An overview of market, migration research, and safety regulations. J. Food Sci., 80, R910-R923 (2015).

Chae, Y. and Y.J. An: Nanoplastic ingestion induces behavioural disorders in terrestrial snails: Trophic transfer effects via vascular plants. Environ. Sci. Nano.,7, 975-983 (2020).

Cheng, Y., R.A. Morshed, B. Auffinger, A.L. Tobias and M.S. Lesniak: Multifunctional nanoparticles for brain tumour imaging and therapy. Adv. Drug Deliv. Rev., 66, 42-57 (2014).

Chouhan, S., H. Swarnkar and S.J.S. Flora: Co-administration of selenium but not iron prevents fluoride toxicity in rats. Biomed. Prev. Nutr., 3, 113-120 (2013).

Clausen, L.P.W. and S.F. Hansen: The ten decrees of nanomaterials regulations. Nature Nanotech., 13, 766-768 (2018).

Cledon, M., S.K. Brar, T.C. Zhang, M. Verma, R.Y. Surampalli and R.D. Tyagi: Nano-ecotoxicology of natural and engineered nanomaterials for microorganisms. In: Nanomaterials in the Environment (Eds.: S.K. Brar, T.C. Zhang, M. Verma, R.Y. Surampalli and R.D. Tyagi). American Society of Civil Engineers, p.652 (2015).

Dai, Y., W. Zhenyu, J. Zhao, L. Xu, L. Xu, X. Yu, Y. Wei and B. Xing: Ethylene mediates $\mathrm{CuO} N \mathrm{NP}$-induced ultrastructural changes and oxidative stress in Arabidopsis thaliana leaves. Environ. Sci.: Nano, 5, 2269-2281(2018).

DBT: Draft 1.Guidelines for evaluation of nano-agriinput products and nano-agriproducts in India. Retrieved from https://dst.gov.in/scientific-programmes/mission-nano-scienceandtechnology-nano-mission (2019).

DS:. HYPERLINK "https://dst.gov.in/sites/default/files/DraftGuidelines\%20.pdf" Io "The pdf file Open in new window" In Draft Guidelines and Best Practices for Safe Handling of Nanomaterials in Research Laboratories and Industries" Nano Mission, DST, Govt of India. Retrieved from https://dst.gov.in/scientificprogrammes/mission-nano-science-andtechnology-nanomission (2019)

DeLoid, G.M., J.M. Cohen, G. Pyrgiotakis and P. Demokritou: Preparation, characterization, and in-vitro dosimetry of dispersed, engineered nanomaterials. Nat Protoc., 12, 355-371 (2017).

Dinesh, R., M. Anandaraj, V. Srinivasan and S. Hamza: Engineered nanoparticles in the soil and their potential implications to microbial activity. Geoderma, 173-174, 19-27 (2012).

Dusinska, M., J. Tulinska, N. Yamani, M. Kuricova, A. Liskova, E. Rollerova, E. Rundén-Pran and B. Smolkova: Immunotoxicity, genotoxicity andepigenetic toxicity of nanomaterials: New strategies for toxicity testing? Food Chem Toxicol., 109, 797-811 (2017) 
Flora, S.J.S.: The Applications, Neurotoxicity, and Related Mechanism of Gold Nanoparticles.In: Neurotoxicity of nanomaterials and nanomedicine. (Eds.: J. Xinguo and H. Gao). Elsevier, USA, pp. 179-203 (2017).

Mittal, M., S. Chatterjee and S.J.S. Flora: Combination therapy with vitamin $\mathrm{C}$ and DMSA for arsenic-fluoride co-exposure in rats. Metallomics, 10, 1291-1306 (2018).

Flora, S.J.S., N. Dwivedi, U. Deb, P. Kushwaha and V. Lomash: Effects of co-exposure to arsenic and dichlorvos on glutathione metabolism, neurological, hepatic variables and tissue histopathology in rats. Toxicol. Res., 3, 23-31 (2013).

García-Gómez, C., S. García, A. Obrador, P. Almendros, D.González and M.D. Fernández: Effect of ageing of bare and coated nanoparticles of zinc oxide applied to soil on the $\mathrm{Zn}$ behaviour and toxicity to fish cells due to transfer from soil to water bodies. Sci Total Environ., 706, 135713 (2020).

DeLoid, G.M., J.M. Cohen, G. Pyrgiotakis and P. Demokritou: Preparation, characterization, and in vitro dosimetry of dispersed, engineered nanomaterials. Nat. Protoc., 12, 355-371 (2017)

Holden, P.A., J.P. Schimel and H.A Godwin: Five reasons to use bacteria when assessing manufactured nanomaterial environmental hazards and fates. Curr. Opin. Biotechnol., 27, 73-78 (2014).

Huang, Y.W., M. Cambre and H.J. Lee: The toxicity of nanoparticles depends on multiple molecular and physicochemical mechanisms. Int. J. Mol. Sci. 18, 2702 (2017).

ISO/TR 12885:2018. Nanotechnologies- Health and safety practices in occupational settings. Technical Report. $2^{\text {nd }} E d n .$, p. 125 (2018).

Jain, A., G.J. Flora, R. Bhargava and S.J. Flora: Influence of age on arsenic-induced oxidative stress in rat. Biol. Trace Elem. Res., 149, 382-390 (2012).

Jain, A., S. Agrawal and S.J.S. Flora: Arsenic and nicotine co-exposure lead to some synergistic effects on oxidative stress and apoptotic markers in young rat blood, liver, kidneys and brain. Toxicol. Rep., 2,1334-1346 (2015).

Kushwaha, P., A. Yadav and S.J.S. Flora: Combinatorial drug delivery strategy employing nano-curcumin and nano-MiADMSA for the treatment of arsenic intoxication in mouse. Chem. Biol. Interact., 286, 78-87 (2018).

Lai, R.W.S, K.W.Y. Yeung, M.M.N. Yung, A.B Djurišić, J.P. Giesy and K.M.Y. Leung: Regulation of engineered nanomaterials: Current challenges, insights and future directions. Environ. Sci. Pollut. Res. Int., 25, 3060-3077 (2018).

Laux, P., J. Tentschert, C. Riebeling, A. Braeuning and O.A. Creutzenberg: Nanomaterials: Certain aspects of application, risk assessment and risk communication. Arch. Toxicol., 92, 121-141 (2018).

Liu, X., W. Li, L. Sun, K.E. Aifantis, B. Yu, Y. Fan, Q. Feng, F. Cui and F. Watari: Effects of physicochemical properties of nanomaterials on their toxicity. J. Biomed. Mater. Res. A., 103, 2499-2507(2015).

$\mathrm{Li}, \mathrm{Y}$. and E. Cummins: Hazard characterization of silver nanoparticles for human exposure routes. J. Environ. Sci. Hlth. Part A, 55,704-725 (2020).

Lowry, G.V., K.B. Gregory, S.C. Apte and J.R. Lead: Transformations of nanomaterials in the environment. Environ. Sci. Technol., 46, 6893-6899 (2012).

Mackay, C.E., M. Johns, J.H. Salatas, B. Bessinger and M. Perri: Stochastic probability modelling to predict the environmental stability of nanoparticles in aqueous suspension. Integr. Environ. Assess. Manage., 2, 293-298 (2006).
Maynard, A.D., P.A. Baron, M. Foley, A.A. Shvedova, E.R. Kisin and V. Castranova: Exposure to carbon nanotubes material: Aerosol release during handling of unrefined single-walled carbon nanotube material. J. Toxicol. Environ. Hlth. A, 67, 87-107 (2004).

Maynard. A.D and R.J. Aitken: Assessing exposure to airborne nanomaterials: Current abilities and future requirements. Nanotoxicology, 1, 26-41 (2007).

Mohiyuddin, S., S. Naqvi and G. Packirisamy: Enhanced antineoplastic/therapeutic efficacy using 5-fluorouracil-loaded calcium phosphate nanoparticles. Beilstein J. Nanotechnol., 9, 2499-2515 (2018).

Murugadoss, S., F. Brassinne, N. Sebaihi, J. Petry, S.M. Cokic, K.L.V. Landuyt, L. Godderis, J. Mast, D. Lison, P.H. Hoet and S.V.D. Brule: Agglomeration of titanium dioxide nanoparticles increases toxicological responses in-vitro and in-vivo. Part Fibre Toxicol., 17, $10(2020)$.

Naqvi, S., M. Samim, M. Abdin, F.J. Ahmed, A. Maitra, C.K. Prashant and A.K. Dinda: Concentration-dependent toxicity of iron oxide nanoparticles mediated by increased oxidative stress. Int. J. Nanomed., 5, 983-989 (2010).

Naqvi, S., A.N. Maitra, M.Z. Abdin, Md. Akmal, I. Arora and Md. Samim: Calcium phosphate nanoparticle mediated genetic transformation in plants. J. Mater. Chem., 22, 3500-3507 (2012).

Naqvi, S., H. Sharma and S.J.S. Flora: Lactobionic acid conjugated quercetin loaded organically modified silica nanoparticles mitigates cyclophosphamide induced hepatocytotoxicity. Int. J. Nanomedi., 14, 8943-8959 (2019).

Naqvi, S., S. Mohiyuddin and P. Gopinath: Niclosamide loaded biodegradable chitosan nanocargoes: An in-vitro study for potential application in cancer therapy. R. Soc. Open Sci., 4, 170611 (2017).

Naqvi, S., S. Mihuyuddin and P. Gopinath: Enhanced antineoplastic / therapeutic efficacy using 5-fluorouracil-loaded calcium phosphate nanoparticles. Beilstein J. Nanotechnol., 9, 2499-2515 (2018).

Naqvi, S., V. Kumar and P. Gopinath: Nanomaterial Toxicity: A challenge to end users. In: Application of Nanomaterials Advances and Key Technologies (Eds.: S. Mohan Bhgyaraj, O.S. Oluwafemi, N. Kalarikal and S. Thomas) Woodhead Publishing, Elsevier Ltd., pp. 315-336 (2018).

Picecchi, D: Tiny Things with a Huge Impact: The International Regulation of Nanomaterials. Mich. J. Envtl. Admin. L., 7, 447 (2018).

Pichardo, S., D. Gutierrez-Praena, M. Puerto, E. Sanchez, A. Grilo and A.M. Camean: Oxidative stress responses to carboxylic acid functionalized single wall carbon nanotubes on the human intestinal cell line Caco-2. Toxicol. In-Vitro, 26, 672-677 (2012).

Panghal, A., H. Sharma, S.J.S. Flora and S. Naqvi: Suicide gene therapy: A promising approach towards gene delivery. Front. Nanosci. Nanotechnol, 5, 1-9, (2019).

Peters, T.M., S. Elzey, R. Johnson, H. Park, V.H. Grassian, T. Maher and P.O. Shaughnessy: Airborne monitoring to distinguish engineered nanomaterials from incidental particles for environmental health and safety. J. Occup. Environ. Hyg., 6, 73-81 (2009).

Sachdeva, S., P. Kushwaha, S.C. Pant, R. Bhargava and S.J.S. Flora: Sodium tungstate induced neurological alterations in rat brain regions and their response to antioxidants. Food Chem. Toxicol., 82,64-72(2015). 
Sharifi, S., S. Behzadi, S. Laurent, M.L. Forrest, P. Stroeve and M. Mahmoudi: Toxicity of nanomaterials. Chem. Soc. Rev., 41, 23232343 (2012).

Sharma, H., S.J.S. Flora and S. Naqvi: Nanomedicine: Anew therapeutic approach in liver diseases. Austin J. Nano. Nanotechnol., 7, 1053 (2019).

Srivastava, D., R.B. Subramanian, D. Madamwar and S.J.S. Flora: Protective value of selenium, calcium and magnesium in arsenic induced oxidative stress in male rats. Arch. Ind. Hyg. Toxicol., 61, 153-159 (2010).

Shrivastava, R., R. Bhargava and S.J.S. Flora: Antioxidant activity and free radical scavenging potential of alpha lipoic acid and quercetin against $\mathrm{Al}_{2} \mathrm{O}_{3}$ nanoparticle-induced toxicity in mice. Free Rad. Antiox., 4, 8-14 (2014a).

Srivastava, R., S. Raza, A. Yadav, P. Kushwaha and S.J.S. Flora: Effects of sub-acute exposure to $\mathrm{TiO}_{2}, \mathrm{ZnO}$ and $\mathrm{Al}_{2} \mathrm{O}_{3}$ nanoparticles on oxidative stress and histological changes in mouse liver and brain. Drug Chem. Toxicol., 37, 336-347 (2014b).

Shrivastava, R., P. Kushwaha, Y.C. Bhutia and S.J.S. Flora: Oxidative stress following exposure to silver and gold nanoparticles in mice. Toxicol. Ind. Hlth., 32,1391-1404, (2016).

Seetharam, R.J. and K.R. Sridhar: Nanotoxicity: Threat posed by nanoparticles. Curr. Sci. 93, 769-770 (2007)

Stone, V. Miller, M.R. Clift, M.J.D. Elder, A. Mills, N.L. Møller, P. Schins, R.P.F. Vogel, U. Kreyling, W.G. Alstrup and K. Jensen: Nanomaterials versus ambient ultrafine particles: An opportunity to exchange toxicology knowledge. Environ. Hlth. Perspect., 125, 106002 (2017).

Quesada-González, D. and A. Merkoçi: Nanomaterial-based devices for point-of-care diagnostic applications. Chem. Soc. Rev., 47, 4697-4709 (2018).

Tsukahara, T. and H. Haniu: Cellular cytotoxic response induced by highly purified multi-wall carbon nanotube in human lung cells. Mol. Cell. Biochem., 352, 57-63 (2011).
Walters, C., E. Pool and V. Somerset: Nanotoxicology: A review. In: Toxicology - New Aspects to This Scientific Conundrum (Eds.: S. Soloneski and M.L. Larramendy). Intech Open. Croatia., pp.45-63 ((2016).

Wang, C., Q. Yang, G. Qin, Y. Xiao and J. Duan: Unveiling a bimetallic $\mathrm{FeCo}$-coupled MoS2 composite for enhanced energy storage. Nanoscale, 12, 10532-10542 (2020).

Wolfram, J., M. Zhu, Y. Yang, J. Shen, E. Gentile, D. Paolino, M. Fresta, G., Nie, C. Chen, H. Shen, M. Ferrari and Y. Zhao: Safety of nanoparticles in medicine. Curr. Drug Targets, 16, 1671-1681 (2015).

Yadav, A., V. Lomash, M. Samim and S.J.S. Flora: Curcumin encapsulated in chitosan nanoparticles: a novel strategy for the treatment of arsenic toxicity. Chem. Biol. Interact., 199, 49-61 (2012).

Yadav, A., R. Mathur, M. Samim, V. Lomash, P. Kushwaha, U. Pathak. A.K. Babbar, S.J.S. Flora, A.K. Mishra and M.P. Kaustuk: Nanoencapsulation of DMSA monoester for better therapeutic efficacy of the chelating agent against arsenic toxicity. Nanomedicine (Lond.), 9, 465-481 (2014).

Yadav, A. and S.J.S. Flora: Nano drug delivery systems: a new paradigm for treating metal toxicity. Expert Opin. Drug Deliv., 13, 831-841 (2016).

Yang, H., C. Liu, D. Yang, H. Zhang and Z. Xi: Comparative study of cytotoxicity, oxidative stress and genotoxicity induced by four typical nanomaterials: The role of particle size, shape and composition. J. Appl. Toxicol., 29, 69-78 (2009).

Yuan, X., X. Zhang, L. Sun, Y. Wei and X. Wei: Cellular toxicity and immunological effects of carbon-based nanomaterials. Part Fibre Toxicol., 16, 18 (2019).

http://dbtindia.gov.in/regulations-guidelines/guidelines-evaluationnano-agri-input-and-nano-agri-products

https://dst.gov.in/scientific-programmes/mission-nano-science-andtechnology-nano-mission 